\title{
Ordering in Lipid Monolayers Studied by Synchrotron X-Ray Diffraction and Fluorescence Microscopy
}

\author{
K. Kjaer and J. Als-Nielsen \\ Risø National Laboratory, DK-4000 Roskilde, Denmark
}

and

C. A. Helm, L. A. Laxhuber, and H. Möhwald

Physics Department E22 (Biophysics), Technische Universität München, D-8046 Garching, West Germany (Received 16 July 1986)

\begin{abstract}
Synchrotron x-ray diffraction results of phospholipid monolayers at an air/water interface are reported and compared with fluorescence microscopic observations of the fluid-gel lipid phase transition. The pressure-induced transition to an orientationally ordered phase occurs at a pressure $\pi_{c}$ which is by more than $15 \mathrm{mN} / \mathrm{m}$ below a pressure $\pi_{s}$ where a transition to a positionally ordered phase occurs. Hence there exists an intermediate phase with long-range orientational and short-range positional order.
\end{abstract}

PACS numbers: $68.10 .-\mathrm{m}, 64.60 .-\mathrm{i}, 87.20 . \mathrm{Cn}$

Lipid monolayers at the air/water interface can be considered as simple model systems for biological membranes. ${ }^{1}$ Structural studies of such model systems are therefore of biological as well as of technological interest, but are also valuable from the more fundamental point of view of structures and phase transitions in two dimensions. ${ }^{2-4}$ The adsorbate density can be varied and measured accurately. Intermolecular forces can additionally be manipulated via water subphase ionic conditions thus affecting surface charge and electrostatic interactions.

Information on structural changes has classically been derived from measurements of the surface pressure as a function of molecular area. ${ }^{5,6}$ Changes in the slopes of the isotherms revealed the existence of phase transitions but the thermodynamic data did not directly yield microscopic information. Two recent experimental developments now make possible a closer analysis of the microstructure of lipid monolayers on water surfaces.

Fluorescence microscopy with dye probes soluble only in the fluid lipid phase allows the fluid-gel phase transition starting at $\pi_{c}$ (see Fig. 1) to be monitored. ${ }^{7-9}$ The light areas represent the fluid phase and the dark areas represent a denser phase which conventionally is called the gel phase (see Fig. 1). ${ }^{10}$

More recently techniques have been developed for the study of liquid surfaces by synchrotron $x$-ray diffraction. ${ }^{11,12}$ By combining this development with the surface $x$-ray diffraction studies of crystalline solid surfaces using glancing incidence near the critical angle for total reflection, ${ }^{13}$ we have been able to obtain diffraction peaks (see Fig. 2) from a lipid monolayer in situ (on water), thus acquiring information about the positional order of the molecules.

Electron diffraction experiments on monolayers transferred onto a solid support have been reported. ${ }^{6}$ The diffraction pattern from a gel-phase domain with a diameter of a few tens of microns is single-crystal type in the sense that it shows distinct spots in hexagonal symmetry. This feature is observed for several substances on a variety of substrates and it seems reasonable that indi-
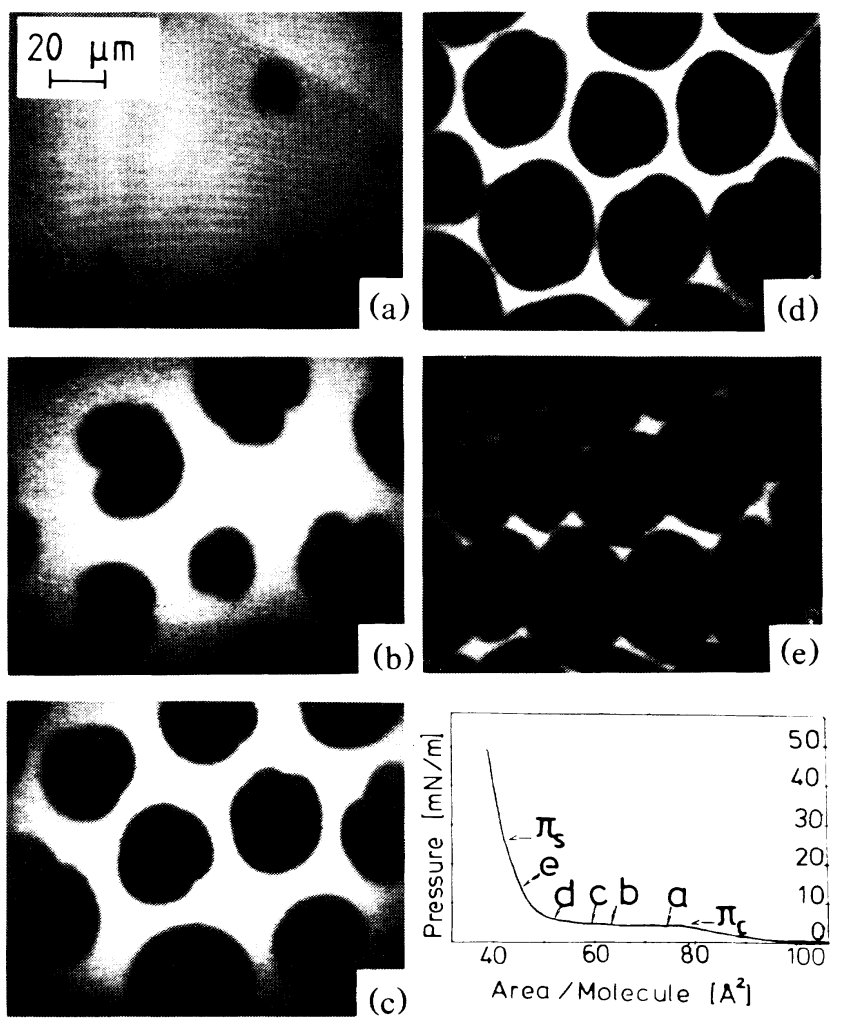

FIG. 1. Fluorescence micrographs of dimyristoylphosphatidic acid monolayer for pressures indicated by arrows in the cor-

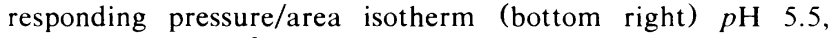
$T=19^{\circ} \mathrm{C}, 5 \times 10^{-5} M$ ethylene diamine tetra-acetic acid. The differences in $\pi_{c}$ and $\pi_{s}$ compared to Fig. 3 are due to a lower ionic strength causing a lower head group charge density. 


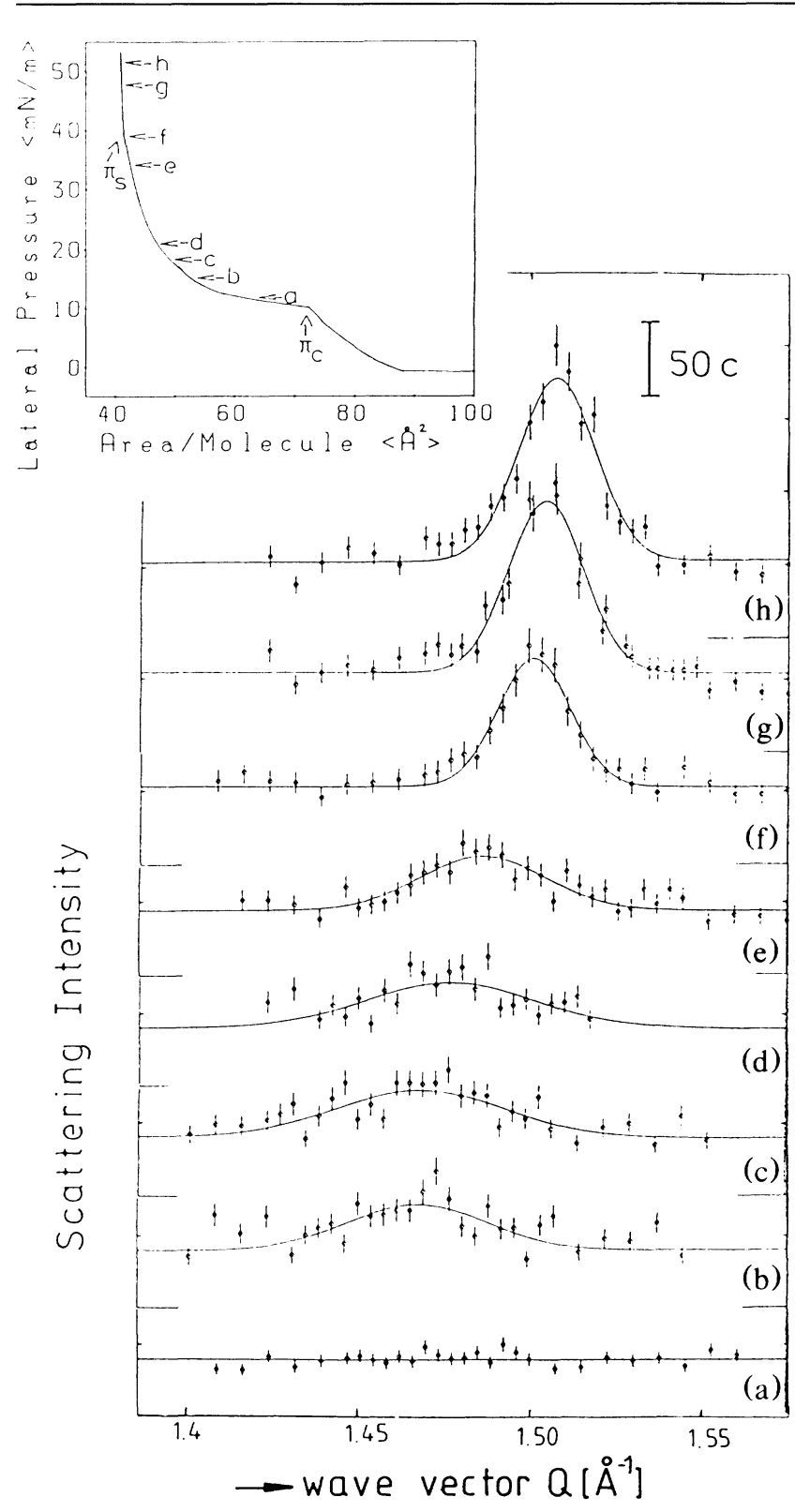

FIG. 2. X-ray intensity as a function of wave-vector transfer $Q$ (bottom) for increasing surface pressures $(a-h)$ corresponding to the arrows marked in the pressure/area isotherm (top). $Q=4 \pi \sin \theta / \lambda$ with $2 \theta$ being the diffraction angle. Dimyristoylphosphatidic acid, $p \mathrm{H} 5.5$, ionic content $10^{-2} M \mathrm{NaCl}$, $5 \times 10^{-5} M$ ethylene diamine tetraacetic acid, $T=19 \pm 2{ }^{\circ} \mathrm{C}$. The lines in the spectra were obtained by fitting of Gaussian curves to the data. The pronounced changes at $\pi_{c}$ and $\pi_{s}$ are also indicated in the isotherm.

vidual gel-phase domains on the surface of water have this property too. ${ }^{14}$ Also, fluorescence polarization studies on similar lipids ${ }^{15}$ are consistent with this assumption. It would therefore be natural to conjecture that the $\mathrm{x}$-ray diffraction patterns in the coexistence region would consist of resolution-limited Bragg peaks with peak intensity decreasing in proportion to the relative amount of dark areas in the fluorescence micrographs.

This is not at all what is observed in Fig. 2. As we shall see in more detail below, the peak width even above $\pi_{s}$ corresponds to an intrinsic finite correlation range of a few hundred angstroms which decreases to only a few tens of angstroms in the coexistence region. A possible solution to this problem is to assume that the gel phase is a hexatic glass as suggested by Nelson, Rubinstein, and Spoepen. ${ }^{16}$ The long range of orientational order of the local crystallographic axes is consistent with the electron diffraction pattern but allows for the short-range order of the positional correlation of the molecules as observed in $x$-ray diffraction.

We shall now give more details about the experiment.

A Wilhelmy-type film balance is placed at the sample position of the $x$-ray diffractometer. A monochromatic $x$-ray beam $(\lambda=1.3815 \AA)$, deflected slightly downwards towards the horizontal liquid surface (the glancing angle being $0.1^{\circ}$ or $75 \%$ of the critical angle for total reflection), is diffracted by the monolayer and powder lines from the two-dimensional powder sample are detected for wave-vector transfer $Q$ of about $1.5 \AA^{-1}$. The width of the peak, measured with a resolution of $0.027 \AA^{-1}$, indicates the correlation range of positional order. Fluorescence microscopic observations were performed with a dedicated film balance containing the objective lens of an invertoscope in the bottom. ${ }^{17}$ Images recorded by a proximity-focus television camera could be transferred to an image-analysis system that (among others) allowed determination of the ratio of areas covered by (dark) domains.

The phospholipid L- $\alpha$-dimyristoylphosphatidic acid (Sigma, Munich) was chromatographically pure and spread from a chloroform/methanol (3:1) mixed solvent. The subphase was distilled and Millipore-filtered water ( $p \mathrm{H} \mathrm{5.5)}$ containing $5 \times 10^{-5} \mathrm{M}$ ethylene diamine tetraacetic acid, tetra sodium salt. Ionic strength was varied by addition of $\mathrm{NaCl}$. The dye probe L- $\alpha$-dipalmitoylnitrobenzoxadiazol-phosphatidylethanolamine used only for the fluorescence microscopic studies applied in a concentration of $1.2 \mathrm{~mol} \%$ was previously shown hardly to affect textures and pressure/area isotherms. ${ }^{18}$

Figure 2 shows a series of $x$-ray diffraction peaks with increasing surface pressure from $a$ to $h$ as indicated in the isotherm at the figure top. The angle corresponding to the peak position monotonically increases with pressure, and concerning linewidth and peak intensity the spectra can be classified into two groups: For pressures below a value $\pi_{s}$ that is clearly defined from a break in the slope of the isotherm (in this case $\pi_{s}=40 \mathrm{mN} / \mathrm{m}$ ) the spectra are weak and broad $(b-e)$. For higher pressures they are more intense and narrower $(f-h)$.

The structural data in Fig. 3 (derived from the diffraction groups in Fig. 2) will now be discussed. The 


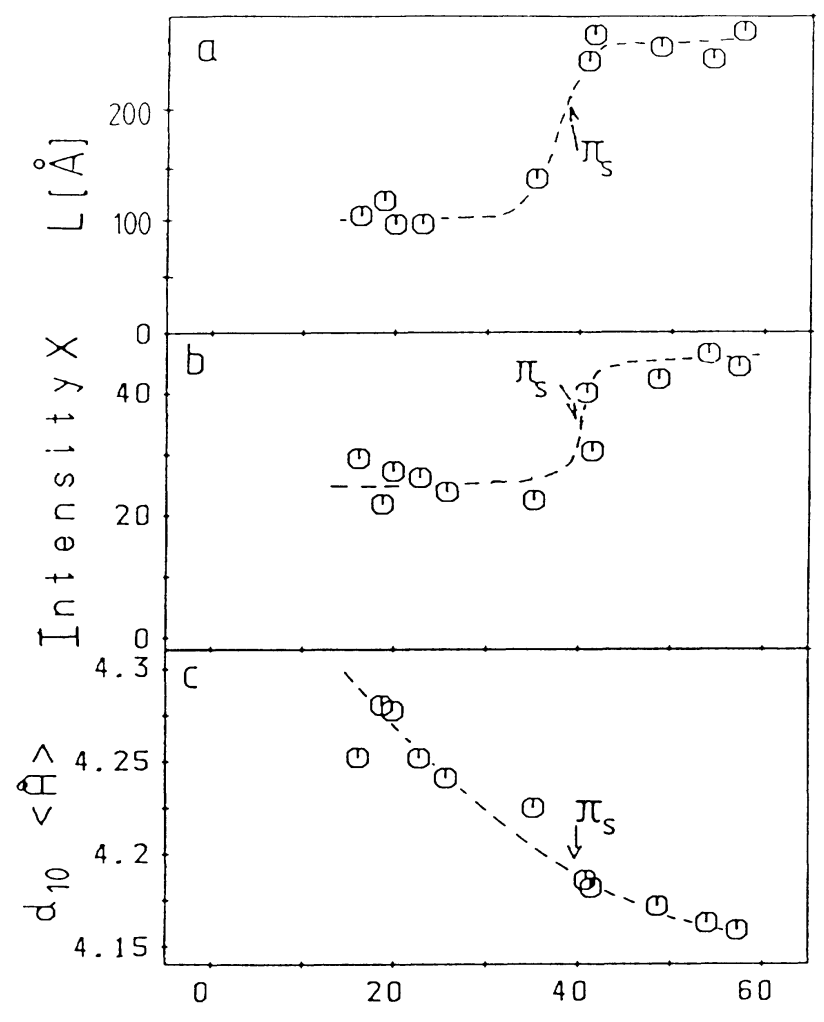

Lateral Pressure $\langle m N / m>$

FIG. 3. (a) Correlation range $L$, (b) integrated intensity $X$, and (c) lattice spacing $d$, as functions of surface pressure for the dimyristoylphosphatidic acid monolayer of Fig. 2. $\pi_{s}$ given in the figure is defined from the slope in the isotherm; $L$ and $d$ are calculated as described in the text. Intensity is given after background subtraction. The broken lines are only guides to the eye.

intrinsic linewidth $[\Delta=\operatorname{HWHM}(Q) / Q]$ was determined from a least-squares fit of the folding of the experimental resolution with an assumed intrinsic Gaussian line shape. ${ }^{19}$ If the intrinsic width is due to a finite size $L$ of crystallites then the relation between $L$ and $\Delta$ is

$$
L=(0.443 / \Delta) d,
$$

where $d$ is the lattice spacing.

Figure 3(a) clearly shows an abrupt change in $L$ from less than $80 \AA$ to more than $250 \AA$. In addition, although there is considerable scatter in the data, the values obtained below and above $\pi_{s}$ are little dependent on pressure within these regimes. The same also holds for the intensity given in Fig. 3(b) where again an abrupt change at $\pi_{s}$ is obvious.

Finally, the bottom part of Fig. 3 shows the lattice spacing $d$ versus pressure. If we assume a hexagonal lattice in accordance with electron diffraction data from Langmuir-Blodgett films, ${ }^{6}$ the lattice spacing $d$ corresponds to the $(1,0)$ planes. As expected qualitatively, $d$

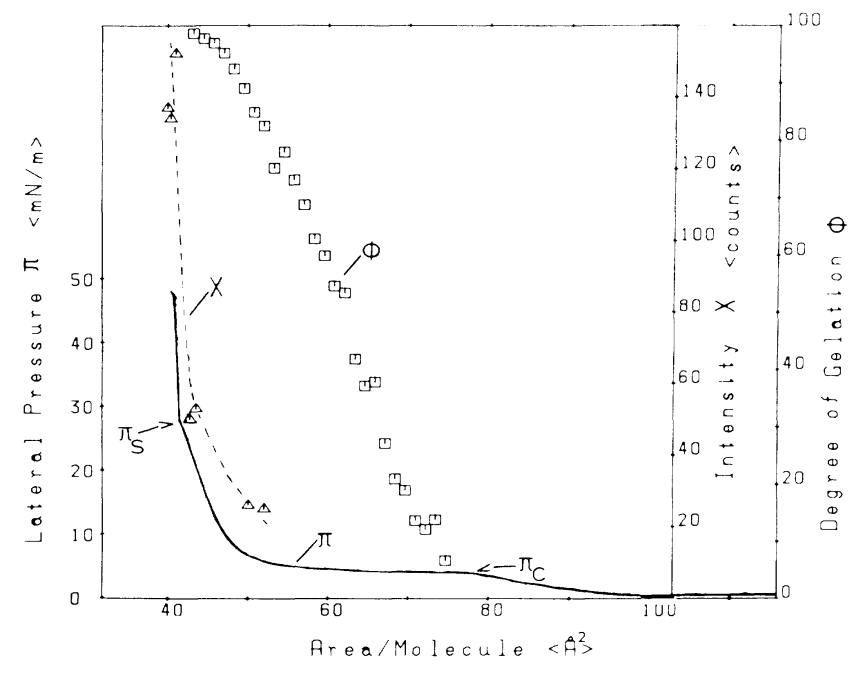

FIG. 4. Degree of gelation $\phi$, calculated as described in the text, $\mathrm{x}$-ray intensity $x$, and surface pressure $\pi$ as functions of molecular area for the monolayer of Fig. 1. The broken line connecting the $x$-ray data is only a guide to the eye.

decreases with increasing pressure but it is not clear whether or not $d$ changes discontinuously at $\pi_{s}$.

The main phase transition beginning at $\pi_{c}$ is followed by fluorescence microscopy. Increasing the surface pressure above $\pi_{c}$, one observes the appearance of gel-phase domains [Fig. 1(a)]. On further compression, these domains grow and exhibit an almost uniform size. They may form a superlattice as a result of long-range repul$\operatorname{sion}^{20,21}$ and for pressures near $\pi_{s}$ the interdomain forces may lead to deformation of domains [Fig. 1(e)].

From the ratio of dark area versus total area in the micrographs one can determine the degree of gelation $\phi$, defined as the fraction of molecules in the gel phase. ${ }^{22} \mathrm{~A}$ linear $\phi$-versus- $A$ relation is obtained in the experiment for $\phi<80 \%$ (Fig. 4). Deviations at higher $\phi$ may be physical effects but may also be due to too large dye enrichment in the remaining fluid phase and will not be discussed further. The intercept of the linear part of the $\phi$-versus- $A$ plot with the abscissa yields $A_{\mathrm{ff}}=80 \AA^{2}$, which is, as expected, close to the mean molecular area at $\pi_{c}$. Extrapolating towards $\phi=1$, one obtains a mean molecular area of $48 \pm 2 \AA^{2}$ in the gel phase coexisting with the fluid, which is significantly larger than that corresponding to $\pi_{s}$ (solid phase). Hence we conclude that the gel phase formed on increase of pressure above $\pi_{c}$ considerably increases in density on approaching $\pi_{s}$.

Comparing $\mathrm{x}$-ray intensity and $\phi$ as a function of molecular area Fig. 4 clearly shows that the two types of experiments respond to different parts in the pressure/area isotherm. This shows that the two transitions leading to translational and orientational order are decoupled. Additional findings described below can further serve to characterize the phase transitions. 
(1) The x-ray line shapes are not yet measured accurately enough for us to draw conclusions regarding the order correlation function. This should enable us to decide if the intermediate phase exists of small solid grains $^{23}$ or is liquid crystalline. ${ }^{3,24,25}$

(2) From the slope of the graph in Fig. 3(c) we derive a decrease in compressibility with increasing pressure. The minimum area compressibility determined from the measured lattice constants at high pressures $\left(\pi>\pi_{s}\right)(7$ $\left.\times 10^{-4} \mathrm{~m} / \mathrm{mN}\right)$ is more than a factor of 2 smaller than the value determined from the pressure/area isotherms. ${ }^{5}$ This means that the latter value is dominated by defect annealing.

(3) The fact that the solid lipid at high pressures is rich in defects also results from the rather broad x-ray lines even in this phase with domain sizes of about 60 times the lattice dimension. This is also consistent with newer observations of about a factor of 4 smaller linewidths under conditions of higher purity control but with identical widths for pressures below $\pi_{s}$.

(4) Concerning kinetic control we could not detect any change in line shape on keeping the film in the solid phase for $3 \mathrm{~h}$. We applied compression speeds between 2 and $5 \AA^{2} /$ molecule min, but could not detect any differences in line shape.

(5) From pressure-area isotherms yielding basically the compressibility, $\kappa$, the transition at $\pi_{s}$ was previously assumed to be second order. ${ }^{5,26}$ To check this we have been concerned with looking for hysteresis on pressure cycling but could not detect it. It is, however, remarkable that the changes at $\pi_{s}$ in coherence length and intensity in Figs. 3(a) and 3(b) are rather abrupt and that we could not detect any measurement point more intermediate between the low- and high-pressure values.

Finally we should mention that these experiments were performed as we varied the surface charge density between 0.35 and $0.9 e^{-}$per molecule. Although the surface charge affected the pressures $\pi_{c}$ and $\pi_{s}$ it did not influence the $x$-ray results as regards coherence length, intensity, and lattice spacing in the two different phases. Similar behavior was also observed with an uncharged lipid. $^{27}$

This work has been funded by the German Federal Minister for Research and Technology under Contract No. 05.366 LA B and by the Danish Natural Science Foundation. We appreciate illuminating discussions with Ole Mouritsen, Martin Zuckermann, and Erich Sackmann.

${ }^{1}$ M. C. Phillips and D. Chapman, Biochim. Biophys. Acta, 163, 301 (1968).
2J. M. Kosterlitz and D. J. Thouless, J. Phys. C 6, 1181 (1973).

${ }^{3}$ D. R. Nelson and B. J. Halperin, Phys. Rev. B 19, 2457 (1979).

${ }^{4}$ D. R. Nelson, Phys. Rev. B 27, 2902 (1983).

${ }^{5} \mathrm{O}$. Albrecht, H. Gruler, and E. Sackmann, J. Phys. (Paris) 39, 301 (1978).

${ }^{6}$ A. Fischer and E. Sackmann, J. Phys. (Paris) 45, 517 (1984).

${ }^{7}$ M. Lösche, E. Sackmann, and H. Möhwald, Ber. Bunsenges. Phys. Chem. 87, 848 (1983).

${ }^{8}$ R. Peters and K. Beck, Proc. Natl. Acad. Sci. U.S.A. 80 , 7183 (1983).

${ }^{9}$ R. M. Weiss and H. M. McConnell, Nature 310, 5972 (1984).

${ }^{10}$ The wording "gel phase" is used throughout this paper for the phases formed at pressure above $\pi_{c}$ without implying any assumptions on the molecular arrangement.

${ }^{11} \mathrm{~J}$. Als Nielsen, F. Christensen, and P. S. Pershan, Phys. Rev. Lett. 48, 1107 (1982).

${ }^{12}$ A. Breslau, M. Deutsch, P. S. Pershan, and J. Bohr, Phys. Rev. Lett. 54, 114 (1985).

${ }^{13}$ P. Eisenberger and W. C. Marra, Phys. Rev. Lett. 46, 1981 (1981).

${ }^{14}$ The $\mathrm{x}$-ray diffraction peak is independent of sample rotation, i.e., powder type rather than single-crystal type. This is understandable since the size of the illuminated area amounts to more than 1000 domains as viewed in the fluorescence micrographs.

${ }^{15} \mathrm{~V}$. Moy, H. E. Gaub, D. Keller, and H. M. McConnell, J. Phys. Chem. 90, 3198 (1986).

${ }^{16}$ D. R. Nelson, M. Rubinstein, and F. Spoepen, Phil. Mag. A 46, 105 (1982).

${ }^{17}$ M. Lösche and H. Möhwald, Rev. Sci. Instrum. 5, 1968 (1984).

${ }^{18}$ M. Lösche and H. Möhwald, Eur. Biophys. J. 1, 35 (1984).

${ }^{19}$ The results concerning the pressure dependence of the linewidth do not depend on the actually assumed line shape. A more detailed measurement and analysis of the latter will be performed in a forthcoming publication.

${ }^{20}$ A. Fischer, M. Lösche, H. Möhwald, and E. Sackmann, J. Phys. (Paris), Lett. 5, L785 (1984).

${ }^{21}$ D. Andelmann, F. Brochard, P. G. de Gennes, and J. F. Joanny, Compt. Rend. 301, 675 (1985).

${ }^{22}$ M. Lösche, Ph.D. thesis, Technische Universität München, 1986 (unpublished).

${ }^{23}$ O. G. Mouritsen and M. J. Zuckermann, Phys. Rev. Lett. 58, 389 (1987).

${ }^{24} \mathrm{G}$. Aeppli and R. Bruinsma, Phys. Rev. Lett. 53, 2133 (1984).

${ }^{25}$ J. Toner and D. R. Nelson, Phys. Rev. B 23, 316 (1981).

${ }^{26}$ D. A. Cadenhead, F. Müller-Landau, and B. M. J. Kellner, in Ordering in Two Dimensions (Elsevier Biomedical, Amsterdam, 1980), pp. 73-81.

${ }^{27} \mathrm{H}$. Möhwald, in The Physics and Fabrication of Microstructures and Microdevices, edited by M. J. Kelly and C. Weisbuch, Springer Series Phys. Vol. 13 (Springer-Verlag, Berlin, 1986). 

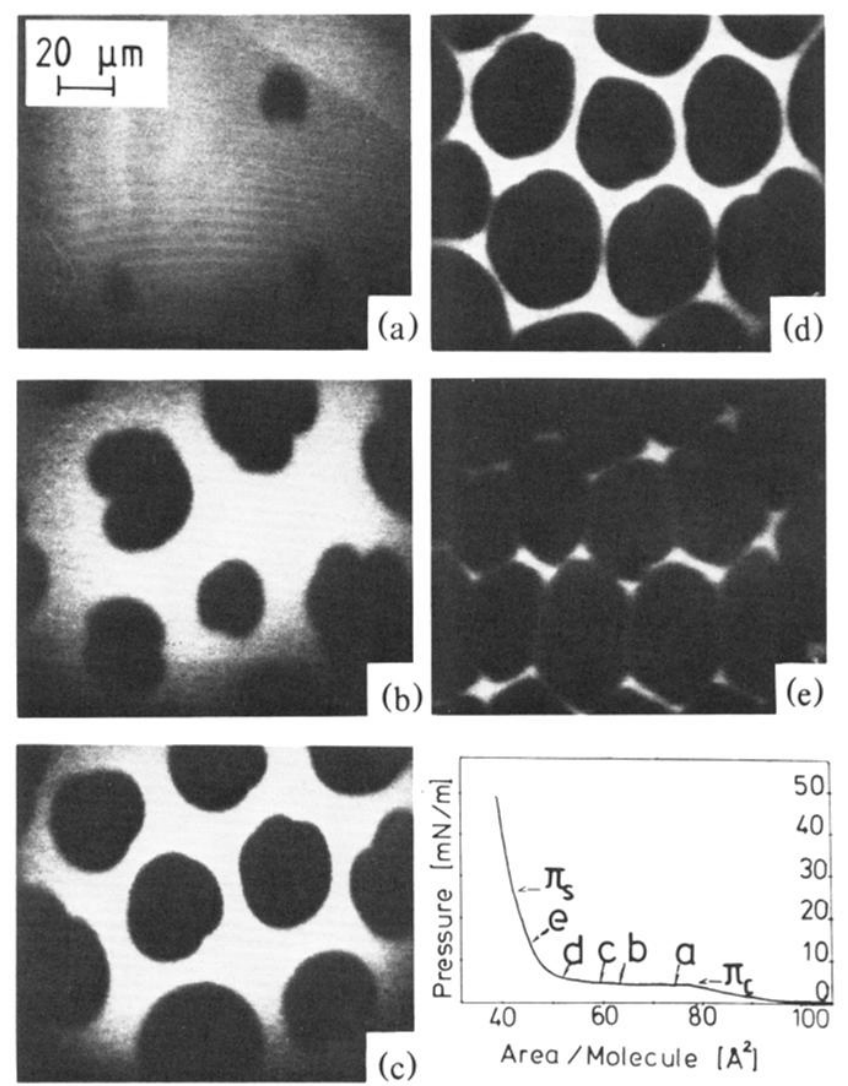

FIG. 1. Fluorescence micrographs of dimyristoylphosphatidic acid monolayer for pressures indicated by arrows in the cor-

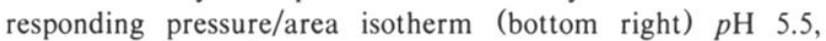
$T=19^{\circ} \mathrm{C}, 5 \times 10^{-5} \mathrm{M}$ ethylene diamine tetra-acetic acid. The differences in $\pi_{c}$ and $\pi_{s}$ compared to Fig. 3 are due to a lower ionic strength causing a lower head group charge density. 